\title{
Robust and Repeatable Biofabrication of Bacteria-Mediated Drug Delivery Systems: Effect of Conjugation Chemistry, Assembly Process Parameters, and Nanoparticle Size
}

\author{
Ying Zhan, Austin Fergusson, Lacey R. McNally, Richey M. Davis, \\ and Bahareh Behkam*
}

Bacteria-mediated drug delivery systems comprising nanotherapeutics conjugated onto bacteria synergistically augment the efficacy of both therapeutic modalities in cancer therapy. Nanocarriers preserve therapeutics' bioavailability and reduce systemic toxicity, while bacteria selectively colonize the cancerous tissue, impart intrinsic and immune-mediated antitumor effects, and propel nanotherapeutics interstitially. The optimal bacteria-nanoparticle (NP) conjugates will carry the maximal NP load with minimal motility speed hindrance for effective interstitial distribution.

Furthermore, a well-defined and repeatable NP attachment density distribution is crucial to determining these biohybrid systems' efficacious dosage and robust performance. Herein, our nanoscale bacteria-enabled autonomous delivery system (NanoBEADS) platform is utilized to investigate the effects of assembly process parameters of mixing method, volume, and duration on NP attachment density and repeatability. The effect of linkage chemistry and NP size on NP attachment density, viability, growth rate, and motility of NanoBEADS is also evaluated. It is shown that the linkage chemistry impacts NP attachment density while the self-assembly process parameters affect the repeatability and, to a lesser extent, attachment density. Lastly, the attachment density affects NanoBEADS' growth rate and motility in an NP sizedependent manner. These findings will contribute to the development of scalable and repeatable bacteria-NP biohybrids for applications in drug delivery and beyond. An interactive preprint version of the article can be found here: https://www.authorea.com/doi/full/10.22541/au.163100509.93917936.

\section{Introduction}

Biohybrid or cell-mediated drug delivery systems, also referred to as biohybrid microrobots, consist of nanocarriers conjugated with host cells, ${ }^{[1-6]}$ attenuated pathogens, ${ }^{[7-9]}$ or generally regarded as safe (GRAS) microorganisms, ${ }^{[10]}$ which are harnessed as living machines for transport of therapeutic loads (for recent comprehensive reviews, see refs. $[11,12])$. In contrast to the traditional systemically administrated therapeutics, biohybrid microrobotic systems for drug delivery are capable of active and targeted delivery using a variety of motility mechanisms (i.e., actuation for self-propulsion) and receptors (i.e., sensors) that enable the cells to process and respond to external signals from their environment, including other cells. The innate actuation and sensing mechanisms powered by chemical energy harvested from the environment provide a distinct advantage to biohybrid microrobots, compared to fully synthetic microrobotic systems that typically rely on external electrical, magnetic, optical, or acoustic sources of energy for controlled actuation. Thus, biohybrid microrobots have considerable potential for targeted delivery of drugs, genes, mRNA, proteins, imaging contrast agents, and radioactive seeds to specific target locations accessible
Y. Zhan, B. Behkam

Department of Mechanical Engineering

Virginia Tech

Blacksburg, VA 24061, USA

E-mail: behkam@vt.edu

The ORCID identification number(s) for the author(s) of this article can be found under https://doi.org/10.1002/aisy.202100135.

(C) 2021 The Authors. Advanced Intelligent Systems published by Wiley$\mathrm{VCH} \mathrm{GmbH}$. This is an open access article under the terms of the Creative Commons Attribution License, which permits use, distribution and reproduction in any medium, provided the original work is properly cited.

DOI: 10.1002/aisy.202100135

\author{
A. Fergusson \\ Virginia Tech \\ Blacksburg, VA 24061, USA \\ L. R. McNally \\ Stephenson School of Biomedical Engineering \\ University of Oklahoma \\ Norman, OK 73019, USA \\ R. M. Davis \\ Department of Chemical Engineering \\ Macromolecules Innovation Institute \\ Virginia Tech \\ Blacksburg, VA 24061, USA
}

Graduate Program in Translational Biology, Medicine, and Health 


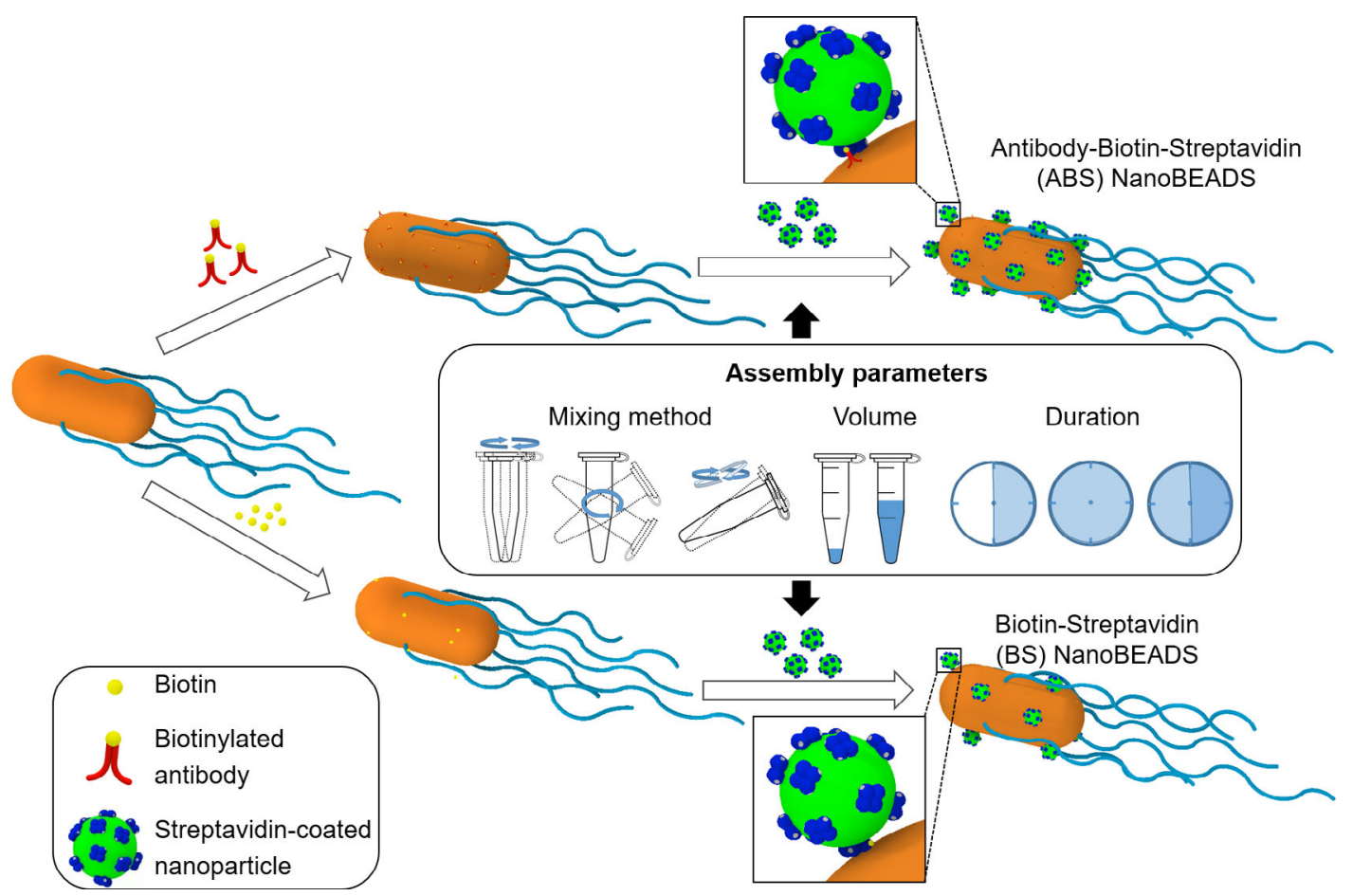

Figure 1. Construction of the NanoBEADS. Schematic of the self-assembly process of two NanoBEADS variants-antibody-biotin-streptavidin NanoBEADS (ABS NanoBEADS) and biotin-streptavidin NanoBEADS (BS NanoBEADS). The conjugation chemistry and assembly process parameters of mixing method, volume, and duration affect the density and repeatability of the NP attachment outcomes.

vascularly, orally, or even interstitially. In particular, bacteria possess unique properties of high speed (up to $\approx 50$ body lengths per second) self-propulsion through blood, ${ }^{[13]}$ mucus, ${ }^{[14]}$ and tissue ${ }^{[15]}$ biased migration or taxis in response to a variety of stimuli (e.g., chemotaxis, ${ }^{[16]}$ aerotaxis, ${ }^{[17]}$ magnetotaxis, ${ }^{[18]}$ phototaxis, ${ }^{[19,20]}$ and $\mathrm{pH}$-taxis ${ }^{[21]}$, and can be genetically manipulated to produce attenuated or auxotrophic strains, ${ }^{[22-24]}$ which support safe and selective colonization of bacteria in vivo. ${ }^{[25-32]}$ Altogether, the advantages of targeted accumulation, deep penetration through self-propulsion, and straightforward genetic manipulation make bacteria an ideal candidate for targeted therapeutic delivery.

Bacteria-based biohybrid drug delivery systems are composed of live bacteria, for sensing and controlled transport, and abiotic micro- or nanoparticles as cargo. Nanocarriers preserve therapeutics' bioavailability and reduce systemic toxicity, while bacteria selectively colonize the cancerous tissue, impart intrinsic and immune-mediated antitumor effects, and propel nanotherapeutics interstitially. Construction of an effective biohybrid drug delivery system requires an attachment mechanism that is stable in vivo and is amenable to interstitial transport, yields sufficient and repeatable cargo attachment density for a predictable therapeutic outcome, and does not render the bacteria nonmotile. Electrostatic interactions, ${ }^{[33,34]}$ hydrophobic interactions, ${ }^{[34]}$ covalent binding, ${ }^{[10,35,36]}$ bioaffinity interactions, ${ }^{[8,37-39]}$ antibody-antigen interactions, ${ }^{[40]}$ or a combination thereof ${ }^{[7,8,39,41]}$ have been used to attach nanoparticles (NPs) to bacteria. Although various attachment methodologies have been explored, a systematic investigation of the effect of the conjugation chemistry and the assembly process parameters on the NP attachment density and repeatability has not been attempted before. Furthermore, the effect of NP load size and quantity on bacterial motility and growth is rarely explored. In this work, we used our previously developed bacteria-based biohybrid platform, known as nanoscale bacteria-enabled autonomous drug delivery system (NanoBEADS) ${ }^{[8]}$ to investigate the aforementioned questions. Two linkage chemistries were separately utilized to attach poly(lactic-co-glycolic acid) (PLGA) NPs to the tumor-targeting Salmonella enterica serovar Typhimurium VNP20009 che $\mathrm{Y}^{+}$bacteria, as shown in Figure 1. The effect of assembly process parameters of mixing method, volume, and duration on the NP attachment density and repeatability was investigated. Subsequently, for the two best performing sets of assembly parameters, the effect of linkage chemistry and NP size on NP attachment density, viability, growth rate, and motility of NanoBEADS was studied. We found the linkage chemistry most significantly affected the NP attachment density. For each of the two binding mechanisms tested, the assembly process parameters also influenced NP attachment's areal density and repeatability. Furthermore, the increase in the NP load-carrying capacity led to an increase in doubling time and a reduction in NanoBEADS motility speed in an NP size-dependent manner.

\section{Results and Discussion}

\subsection{Effect of the Assembly Process Parameters on NP Attachment Density}

The NP attachment density is critical to the efficacy of bacteriabased drug delivery systems, as it determines the amount of 
Table 1. Table of assembly process parameters (see Methods for details).

\begin{tabular}{lccc}
\hline Mixing method & $\begin{array}{c}\text { Speed } \\
{[\mathrm{rpm}]}\end{array}$ & $\begin{array}{c}\text { Assembly } \\
\text { volume }[\mu \mathrm{L}]\end{array}$ & $\begin{array}{c}\text { Assembly } \\
\text { period }[\mathrm{min}]\end{array}$ \\
\hline Vortex mixer (V) & 500 & 100 & $30,60,90$ \\
Vortex mixer (V) & 500 & 800 & $30,60,90$ \\
Belly Dancer mixer (B) & 100 & 800 & $30,60,90$ \\
End-over-end mixer (E) & 15 & 800 & $30,60,90$ \\
\hline
\end{tabular}

therapeutic load carried by each NanoBEADS agent. We first investigated the effect of mixing method (i.e., the type of mechanical mixer employed when mixing the streptavidin-coated NPs and the biotinylated bacterial suspensions, Video S1, Supporting Information), assembly volume (i.e., total suspension volume during mixing), and assembly period, as depicted in Table 1 , on the formation of antibody-biotin-streptavidin NanoBEADS (ABS NanoBEADS) using $165 \pm 11 \mathrm{~nm}$ diameter NPs. Informed by our prior work, ${ }^{[9]}$ all NanoBEADS assembly experiments were conducted at the bacteria to NPs ratio 1:100 and biotinylated antibody concentration of $10 \mu \mathrm{g} \mathrm{mL}^{-1}$ (see Methods for detail). The number of attached NPs on each bacterium was quantified using scanning electron microscopy (SEM) and reported as the number of attached NPs per projected unit area of the cell surface (\#NPs $\mu \mathrm{m}^{-2}$ ).

We first evaluated the effect of assembly volume and duration using a vortex mixer $(\mathrm{V})$ for two different assembly volumes of $100 \mu \mathrm{L}(\mathrm{V}-100)$ and $800 \mu \mathrm{L}(\mathrm{V}-800)$ and three different assembly periods of 30, 60, and 90 min. As shown in Figure 2a, assembly volume had no statistically significant effect on the average NP attachment density of ABS NanoBEADS constructed using a vortex mixer, except for the significantly decreased NPs attachment density in the $60 \mathrm{~min}$ assembly period, when the volume increased from 100 to $800 \mu \mathrm{L}(p<0.01)$. In the case of $100 \mu \mathrm{L}$ assembly volume, the NPs attachment density significantly increased by increasing duration from 30 to $60 \mathrm{~min}$ $(p<0.01)$. In contrast, there was no significant difference in the NPs attachment density for V-800-60 comparing to the other two durations of V-800, as shown in Figure 2a,b. Keeping the high throughput biofabrication needs for in vivo experiments and future translational applications in mind, we examined (a)

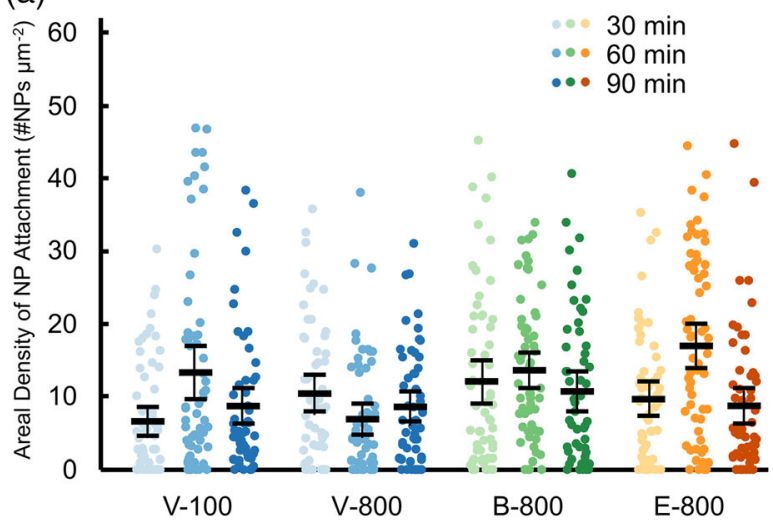

(c)

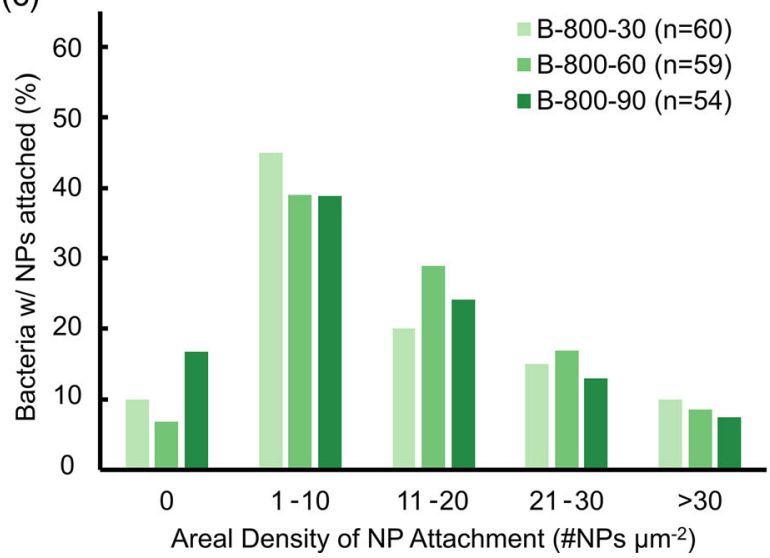

(b)

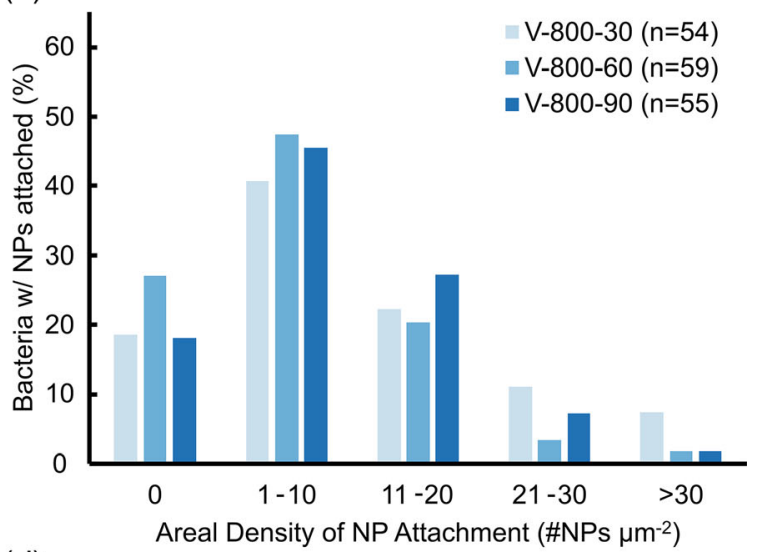

(d)

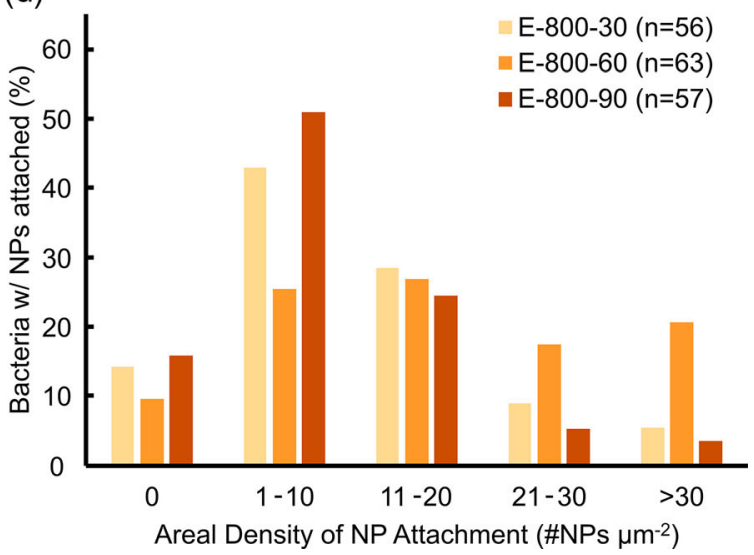

Figure 2. Effect of assembly process parameters on $165 \mathrm{~nm}$ diameter NP attachment density in ABS NanoBEADS. a) Effect of mixing method, assembly volume, and assembly duration on the attached NP areal density. The black bars indicate means and $95 \%$ confidence intervals (i.e., nonoverlapping bars between any two cases indicate a significant difference with a $p<0.05)$. Effect of assembly period on the distribution of the attached NP areal density for b) V-800, c) B-800, and d) E-800. In all plot legends, X-000-00 denote the mixing method (X), assembly volume (000), and assembly period (00), as described in Table 1. All areal densities are calculated based on projected cell area. 
the other two mixing methods at the higher $800 \mu \mathrm{L}$ volume. We then compared the results from V-800 with those of the Belly Dancer mixer (B-800) and the end-over-end mixer (E-800) for each of the three assembly durations.

For all the assembly periods tested with the $800 \mu \mathrm{L}$ volume, the Belly Dancer and the end-over-end mixer resulted in similar or higher average NPs areal density than the vortex mixer (Figure 2a). Furthermore, the vortex mixer resulted in the lowest count of NanoBEADS with attachment density higher than $10 \mathrm{NPs} \mu \mathrm{m}^{-2}$ cell area and the highest count of bacteria with no NP attachment (Figure $2 \mathrm{~b}-\mathrm{d}$ ). Thus, we eliminated the vortex mixer. For the other two mixing methods, as the assembly period increased from 30 to $60 \mathrm{~min}$, the count of NanoBEADS with attachment numbers higher than $10 \mathrm{NPs} \mu \mathrm{m}^{-2}$ cell area showed an appreciable increase while the number of bacteria without any $\mathrm{NP}$ attached $\left(0 \mathrm{NPs} \mu \mathrm{m}^{-2}\right)$ decreased (Figure $\left.2 \mathrm{c}, \mathrm{d}\right)$. Upon increasing the assembly time to $90 \mathrm{~min}$, more NanoBEADS with attachment density lower than $10 \mathrm{NPs} \mu \mathrm{m}^{-2}$ were observed (Figure 2c,d), resulting in an overall decrease in average attachment density (Figure 2a). This reduction in attachment density can be attributable to the presence of residual culture medium in the assembly volume, which led to some bacterial growth during the more extended assembly period. Therefore, $60 \mathrm{~min}$ was identified as the optimal assembly duration. B-800-60 and E-800-60 cases had significantly higher NPs attachment density than V-800-60 case $(p<0.05)$. Additionally, there was a significant difference in the NPs attachment density in E-800-60 comparing to the other two durations $(p<0.01)$. Moreover, the E-800-60 case had a statistically significant higher average NP attachment density than the NanoBEADS constructed using a vortex mixer, except for the V-100-60 case, where the difference was not statistically significant. Altogether, our results suggest that the Belly Dancer mixer used with $800 \mu \mathrm{L}$ assembly volume for $60 \mathrm{~min}$ (B-800-60) and the end-over-end mixer used with $800 \mu \mathrm{L}$ assembly volume for $60 \mathrm{~min}$ (E-800-60) were the optimal sets of assembly parameters, among the combinations tested.

\subsection{Effect of the Linkage Chemistry on Areal Density and Repeatability of NP Attachment}

Due to the stochastic nature of the assembly process, a distribution of the particle attachment density is expected for each individual experiment, as shown in Figure 2. However, minimizing the variance in NP cargo attachment density distribution across replicate experiments is crucial to determining the efficacious dosage of biohybrid systems toward robust and predictable therapeutic outcomes. Thus, we next examined the repeatability in NP attachment density outcomes for two linkage chemistries using the optimal assembly parameters of B-800-60 and E-800-60. In both NanoBEADS variants, streptavidin-coated NPs were attached to the bacteria using the high affinity biotin-streptavidin interactions; however, in the ABS NanoBEADS variant, biotin was conjugated with antiSalmonella antibody and covalently bonded to the antigen on bacteria, whereas in the biotin-streptavidin NanoBEADS (BS NanoBEADS) variant, biotin was adsorbed onto the bacteria via electrostatic interactions. A minimum of four independent replicate assembly experiments for each of the two NanoBEADS variants were conducted (Figure 3). For the ABS NanoBEADS, both B-800-60 and E-800-60 yielded similar results (Figure 3a, b), with E-800-60 producing a higher fraction of high NP density NanoBEADS (>20 (\#NPs $\mu \mathrm{m}^{-2}$ ) (Figure $3 \mathrm{c}$ ) and a more repeatable attachment density distribution with a smaller variance (Figure 3d). Thus, we identify E-800-60 as the optimal set of assembly parameters for producing the ABS NanoBEADS. For the BS NanoBEADS, no significant difference between the B-800-60 and E-800-60 outcomes was observed (Figure 3e,f). Comparing the distribution of the attached NP areal density between the two variants (Figure $3 \mathrm{c}, \mathrm{g}$ ), it is evident that the ABS NanoBEADS had a significantly higher number of bacteria with greater attachment density than the BS NanoBEADS. Furthermore, only $\approx 7 \%$ of the bacteria did not have any NP attached when the ABS linkage is used, whereas an average of $\approx 27 \%$ did not have any NP attached when the BS linkage is used. Consistent with our observation for ABS NanoBEADS, E-800-60 produced a more repeatable attachment density distribution with a smaller variance (Figure $3 \mathrm{~h}$ ). The viability of the bacteria was not affected by the attached NPs in either of the two variants (Figure S1, Supporting Information).

\subsection{Effect of NP Size on Attachment Density and Growth Rate}

Next, we investigated the effect of particle size on the attachment density outcome in the ABS and BS NanoBEADS variants constructed using the optimal assembly parameters of E-800-60 and $165 \pm 11 \mathrm{~nm}$ or $121 \pm 6 \mathrm{~nm}$ particles. Representative SEM images of both NanoBEADS variants are shown in Figure 4a-d. As shown in Figure 4e, NPs with a smaller diameter of $121 \pm 6 \mathrm{~nm}$ had significantly increased attachment density $(p<0.0001)$ for ABS NanoBEADS, but the slightly increased attachment density for BS NanoBEADS was not statistically significant. Consistent with our observation of NanoBEADS constructed with large NPs, there was an average of $70 \%$ decrease in the NP areal density upon changing from the ABS linkage chemistry to the antibody-free BS linkage chemistry (Figure 4e). Our results suggest that irrespective of NP size, the linkage chemistry (i.e., the method used to present biotin on the bacteria outer membrane) significantly affects the NP attachment outcome. When the biotinylated antibody is used, more particles per bacteria are attached compared to using physisorbed biotin. The observed trends in the effect of NP size on the NP attachment density were conserved when the other set of optimal assembly parameters (B-800-60) were used (Figure S3a, Supporting Information).

We next examined the effect of NP size and attachment density on the growth of NanoBEADS. While NanoBEADS can be stored in media that do not support growth in vitro, they are expected to operate in vivo for days ${ }^{[9]}$ and are observed to grow in the nutrient-rich tumor microenvironment. ${ }^{[42]} \mathrm{As}$ NanoBEADS grow, the attached NP load is divided among the daughter cells, leading to a more effective distribution of the NPs within the tumor. ${ }^{[9]}$ Furthermore, many tumor-targeting bacteria, including $S$. Typhimurium VNP20009 $\mathrm{cheY}^{+}$used in this study, have intrinsic and immune-mediated antitumor 
(a)

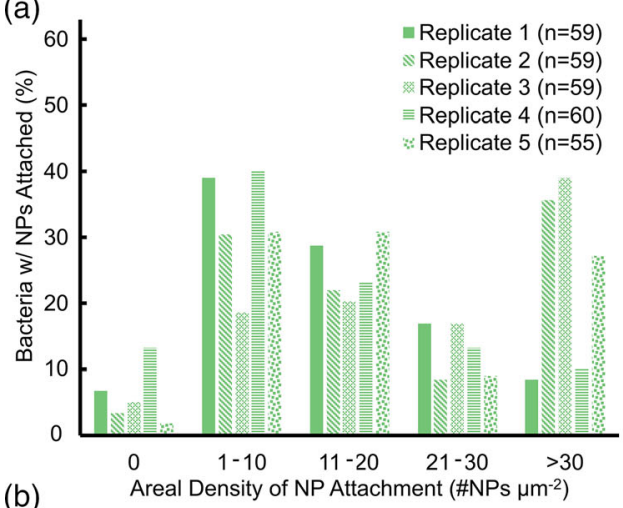

(b)

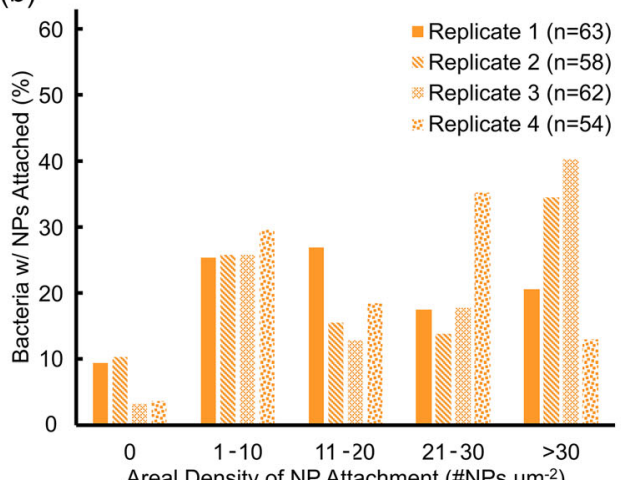

(c)
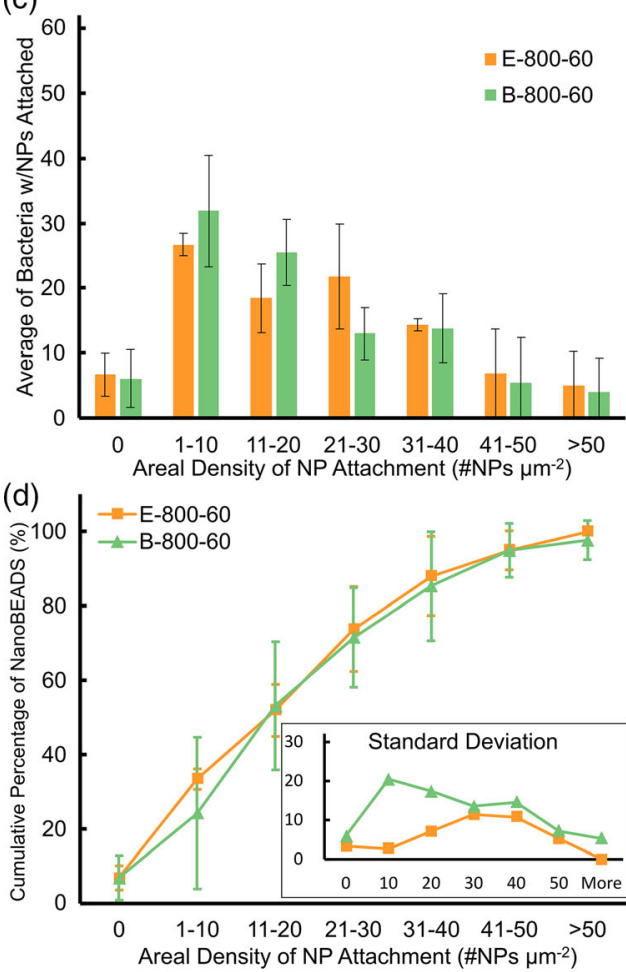

(e)

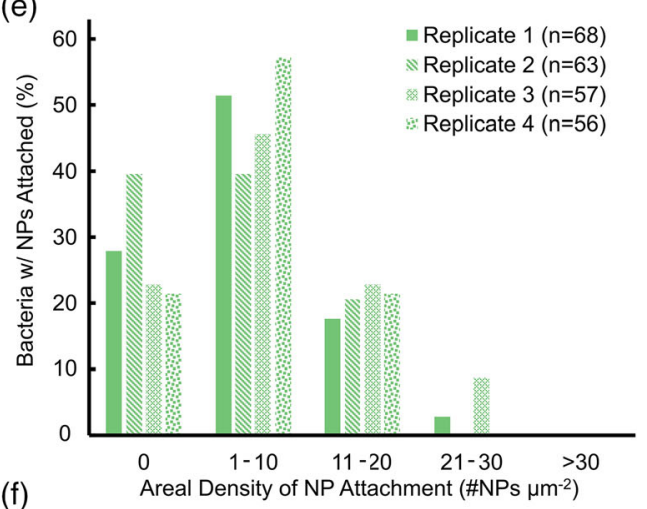

(f)

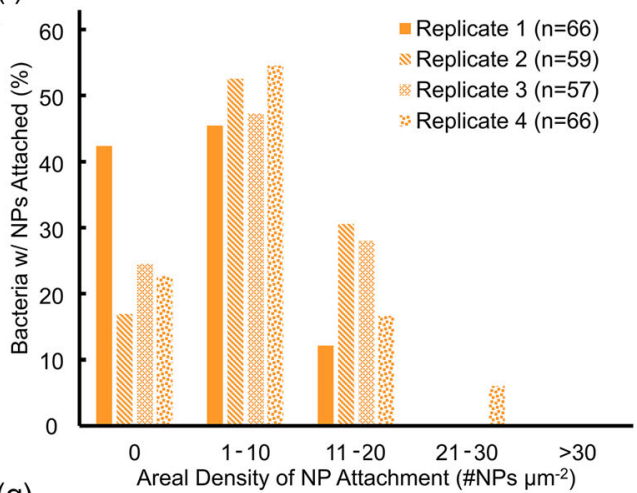

(g)

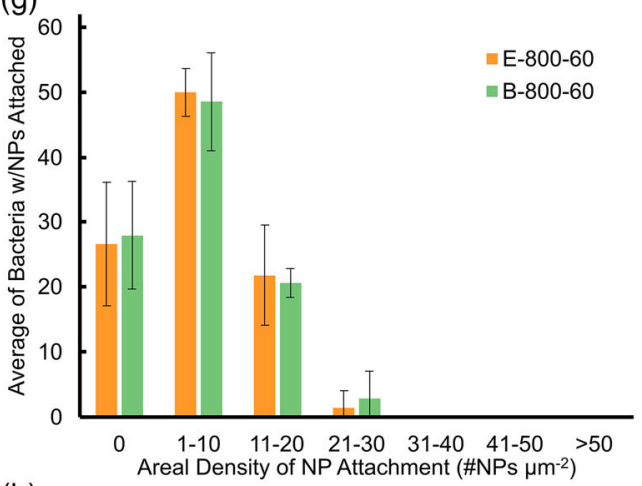

(h)

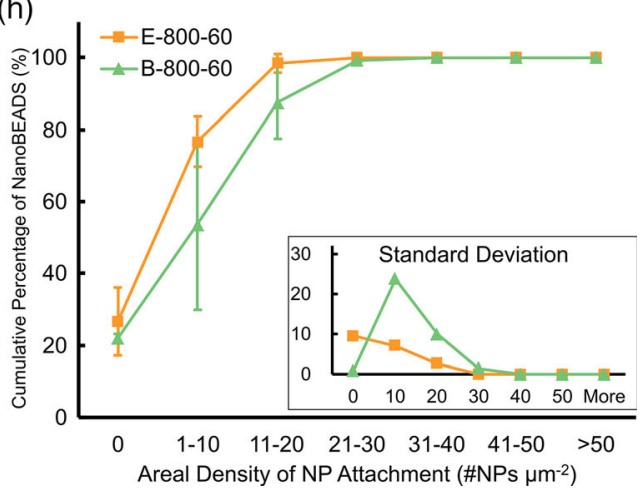

Figure 3. Effect of mixing method and linkage chemistry on the areal density and repeatability of NP attachment. Repeatability of NP attachment areal density distribution for ABS NanoBEADS constructed using the process parameters of a) B-800-60 and b) E-800-60, and for BS NanoBEADS constructed using process parameters of e) B-800-60 and f) E-800-60. Average NP attachment areal density distribution for c) ABS NanoBEADS and g) BS NanoBEADS constructed using the process parameters of B-800-60 and E-800-60. Cumulative percentage of NanoBEADS and standard deviation as a function of NP areal density (inset) for d) ABS NanoBEADS and h) BS NanoBEADS constructed using the process parameters of B-800-60 and E-800-60. X-000-00 denotes mixing method $(X)$, assembly volume (000), and assembly period (00), as described in Table 1 . All areal densities are calculated based on projected cell area. All error bars are standard deviations. 


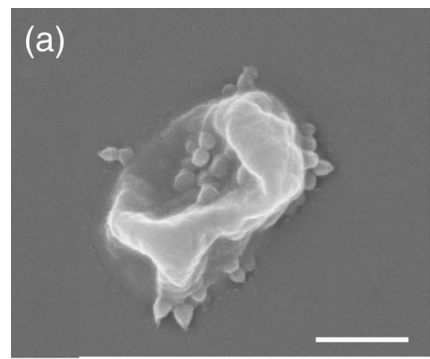

(e)

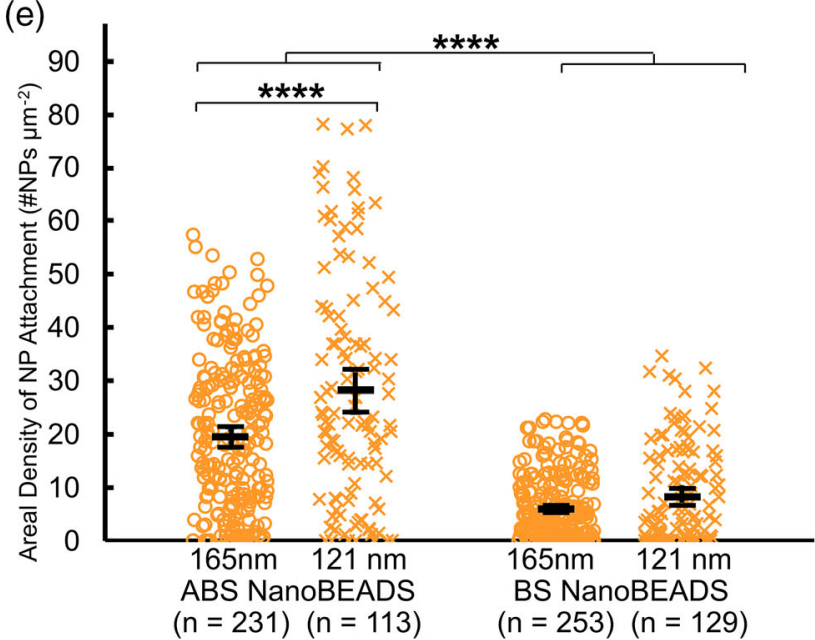

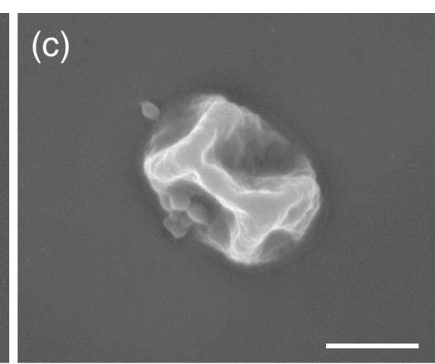

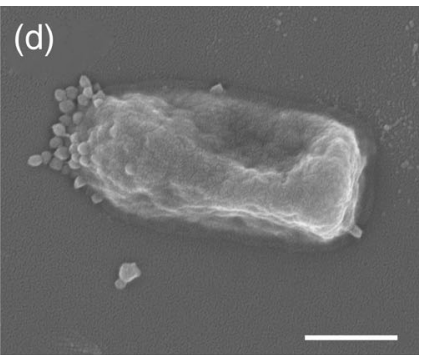

(f)

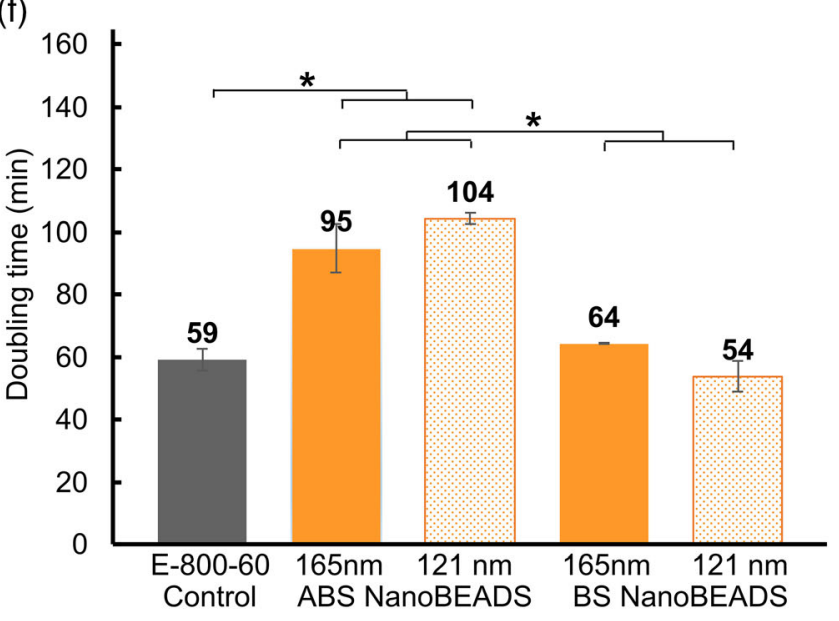

Figure 4. Effect of NP size and linkage chemistry on attachment density and growth rate. Representative SEM images of a) ABS NanoBEADS (E-800-60) with $165 \mathrm{~nm}$ NPs, b) ABS NanoBEADS (E-800-60) with $121 \mathrm{~nm}$ NPs, c) BS NanoBEADS (E-800-60) with $165 \mathrm{~nm}$ NPs, d) BS NanoBEADS (E-800-60) with $121 \mathrm{~nm}$ NPs. All scale bars are $500 \mathrm{~nm}$. e) The NP areal attachment density as a function of NP size for the ABS NanoBEADS and BS NanoBEADS variants. The black bars indicate means and $95 \%$ confidence intervals. ${ }^{*} *{ }^{\prime} * p<0.0001$. f) The comparison of doubling time of the variants of NanoBEADS and mechanically treated (E-800-60) bacteria with no particles attached as the control. Each data point represents the mean \pm standard deviation. ${ }^{*} p<0.05$.

effects $^{[43,44]}$ in addition to serving as a delivery vector for the nanomedicine attached to their outer membrane. Thus, intratumoral bacterial growth augments the therapeutic effect of fixed nanomedicine payload. The doubling times of both NanoBEADS variants at both NP sizes were measured and compared to the doubling time of the bacteria (control). All experiments were performed in a mammalian cell culture medium, given the ultimate application of the NanoBEADS platform as a cancer drug delivery system. For the optimal assembly parameters, i.e., E-800-60, we first investigated if coating of the outer membrane or the mechanical agitation of the assembly process affects the growth rate. We found no statistically significant difference in the average doubling time of unmodified bacteria, biotinylated antibody-coated bacteria, and biotin-coated (physisorbed) bacteria, suggesting that surface modification did not affect the bacterial growth rate (Figure S2, Supporting Information). However, bacteria subjected to mechanical agitation experienced during the E-800-60 assembly process (in the absence of NPs) resulted in a statistically significant $(p<0.05)$ increase in the average doubling time of the bacteria from $49 \pm 3 \mathrm{~min}$ to $59 \pm 4 \mathrm{~min}$, which may be in part attributable to the mechanical stress. Next, we measured the doubling time of the NanoBEADS constructs and compared them to the doubling time of the mechanically treated (E-800-60) bacteria as the baseline control
(Figure 4f). The average doubling time of the 165 and $121 \mathrm{~nm}$ ABS NanoBEADS was $95 \pm 8$ and $104 \pm 2 \mathrm{~min}$, both of which were significantly longer $(p<0.05)$ than the control doubling time. The average doubling time of the 165 and $121 \mathrm{~nm}$ BS NanoBEADS was significantly shorter $(p<0.05)$ than their ABS NanoBEADS counterparts at $64 \pm 0.2$ and $54 \pm 5 \mathrm{~min}$, respectively. Interestingly, no significant difference between the doubling time of BS NanoBEADS and control bacteria was observed. We attribute the significantly longer doubling time of the ABS NanoBEADS $(p<0.05)$ to the higher NP attachment areal density, as shown in Figure 4a. NP size did not significantly affect the NanoBEADS doubling time within each variant type $(p>0.05)$. Altogether, our results suggest that NP attachment density and not the NP size affects the NanoBEADS doubling time for the range of NP size tested. The observed trend in the effect of NP size on growth rate was conserved when the closely comparable B-800-60 assembly parameters were used (Figure S3b, Supporting Information). Lastly, we note that the dependency of doubling time on NP attachment density may become less significant with particle size reduction. We observed that the doubling time of the ABS NanoBEADS constructed with significantly smaller $40 \mathrm{~nm}$ gold particles at comparable NP attachment density to $165 \mathrm{~nm}$ PLGA particles was comparable to that of control bacteria (Figure S4 and S5, Supporting Information). 
(a)

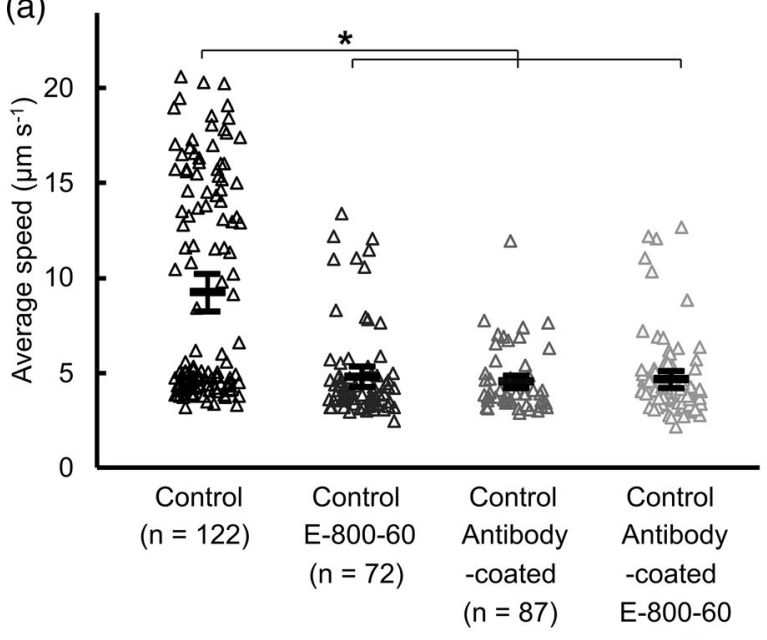

(b)

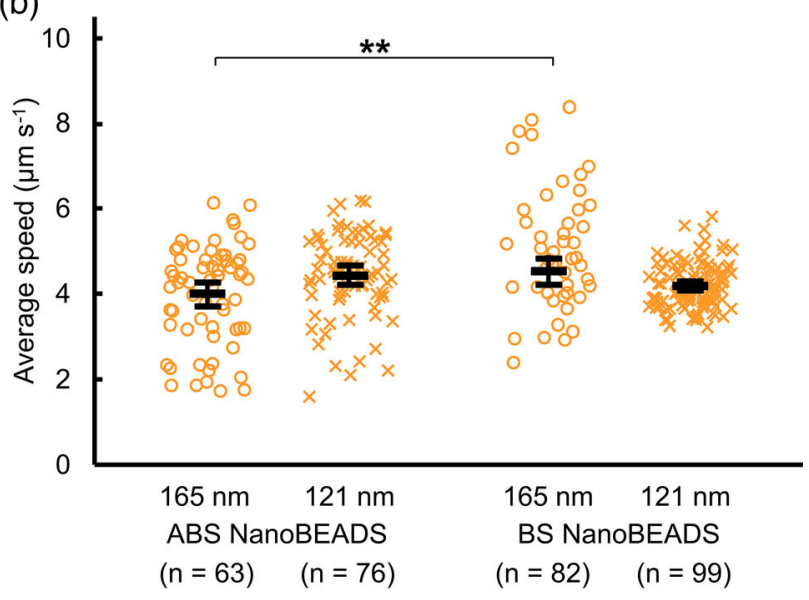

Figure 5. Effect of NP size, quantity, and linkage chemistry on NanoBEADS motility speed. a) The effect of mixing-induced shear and antibody coating on the average speed of bacteria (without NPs) at $2 \mathrm{~h}$ incubation time. b) The average speed of the ABS and BS NanoBEADS constructed using small or large $\mathrm{NPs}$ at $2 \mathrm{~h}$ incubation time. In both plots, the black bars indicated means and $95 \%$ confidence intervals. $* p<0.05 ; * * p<0.01$.

\subsection{Effect of NP Size and Attachment Density on NanoBEADS Motility}

We next assessed the effect of NP size and attachment density on motility by measuring each NanoBEADS variant's swimming speeds with small or large NP attached and compared the speeds with that of bacteria as the baseline (control). Before characterizing NanoBEADS motile behavior, we first evaluated how chemi$\mathrm{cal}$ and mechanical processes that comprise the NanoBEADS construction process affect the bacteria motility in the absence of NPs. The average speeds of the bacteria cultured under standard microbiological conditions (control), mechanically treated bacteria without antibody coating or NP attachment (control, E-800-60), antibody-coated bacteria, and mechanically treated (E-800-60) antibody-coated bacteria without particles are shown in Figure 5a. Mechanical agitation decreased the average speed of bacteria from $9.2 \pm 5.6$ to $4.8 \pm 3.9 \mu \mathrm{m} \mathrm{s}^{-1} \quad(p<0.05)$. However, the choice of mixing method did not have a statistically significant effect on the bacteria motility speed (Figure S3c, Supporting Information). Antibody coating of bacteria had a similar statistically significant reducing effect on the bacteria motility speed to $4.5 \pm 1.5 \mu \mathrm{m} \mathrm{s}^{-1}(p<0.05)$. The combination of mechanical agitation and antibody coating did not result in any further statistically significant degradation in speed $\left(4.7 \pm 2.1 \mu \mathrm{m} \mathrm{s}^{-1}\right)$. We attribute the reduction in swimming speed to the fragile structure of the flagella in S. Typhimurium VNP20009 $\mathrm{cheY}^{+}$, which can be disrupted through mechanical agitation. ${ }^{[45]}$

We next examined the motility speed of both NanoBEADS variants constructed using the E-800-60 assembly parameters and attached with 165 or $121 \mathrm{~nm}$ NPs, as shown in Figure 5b and S3d, Supporting Information. To assess the effect of NP size, we compared the motility speed within each variant category. We did not observe a statistically significant difference between the $121 \mathrm{~nm}$ ABS NanoBEADS average swimming speed $\left(4.4 \pm 1.0 \mu \mathrm{m} \mathrm{s}^{-1}\right)$ and the $165 \mathrm{~nm}$ ABS NanoBEADS $\left(4.0 \pm 1.2 \mu \mathrm{m} \mathrm{s}^{-1}\right)$. It appears that the contribution of the larger $165 \mathrm{~nm}$ NPs to the drag force is comparable to the contribution of the larger quantity of the smaller $121 \mathrm{~nm}$ particles (Figure $4 \mathrm{e}$ ). In the case of BS NanoBEADS, the NanoBEADS with the smaller $121 \mathrm{~nm}$ NPs have a lower average speed $\left(4.2 \pm 0.5 \mu \mathrm{m} \mathrm{s}^{-1}\right)$ than NanoBEADS with $165 \mathrm{~nm}$ NPs $\left(4.5 \pm 1.4 \mu \mathrm{m} \mathrm{s}^{-1}\right)$, but the difference is not statistically significant. To assess the effect of linkage chemistry on motility speed, we compared the motility speed of the two NanoBEADS variants at each particle size. In the case of NanoBEADS with $165 \mathrm{~nm}$ NPs, BS NanoBEADS have a significantly higher average speed than ABS NanoBEADS $(p<0.01)$, presumably due to the lower NP attachment density. Whereas, for the NanoBEADS with the smaller $121 \mathrm{~nm} \mathrm{NPs,} \mathrm{both}$ NanoBEADS variants have similar average speed, which was not significantly different from the average speed of antibodycoated E-800-60 control bacteria.

\section{Conclusion}

The NanoBEADS is a bacteria-based biohybrid drug delivery system designed to utilize self-propelling motile bacteria to enhance the interstitial delivery of nanomedicine. As is the case for all biohybrid delivery systems, the effectiveness of NanoBEADS hinges upon maximizing its NP load without affecting its viability and minimal effect on its motility. It is also imperative that the NanoBEADS construction process produces a repeatable distribution of NP load densities (i.e., therapeutic load) to facilitate the use of such a system in translational applications. Thus, a systematic investigation of the effects of the assembly process parameters, linkage chemistry, and NP size on NanoBEADS properties (i.e., NP attachment density and repeatability, growth rate, and swimming speed) was carried out. We selected biotin-streptavidin linkage chemistry for this study due to its prevalence in constructing bacteria-based biohybrid microrobots. $^{[7,8,37-39,41,46-50]}$ Furthermore, the peritrichously 
flagellated $S$. Typhimurium was selected due to the common use of Gram-negative bacteria (Escherichia coli, Serratia marcescens, etc.) as well as other flagellated bacteria in the construction of bacteria-based microrobots. The selected PLGA NPs of two different sizes represent a good model for the polymeric NPs used in constructing biohybrid microrobots. Using an end-over-end mixer with a total mixing volume of $800 \mu \mathrm{L}$ for $60 \mathrm{~min}$ assembly period (E-800-60) produced the highest and most repeatable (i.e., smallest variance) NP attachment density without affecting the viability of the bacteria. For BS linkage chemistry, where streptavidin-functionalized NPs were attached to the bacteria using biotin that was physisorbed to the bacteria, the attachment density decreased by more than $70 \%$, compared to the ABS linkage chemistry case. The optimal NanoBEADS construction strategy reported herein is extendible to other NP materials and sizes (Figure S4, Supporting Information). The motility speed is adversely affected with the attachment of larger particles at high density, but the attachment of NPs smaller than $120 \mathrm{~nm}$ did not affect the motility speed even at a high attachment density (Figure S5, Supporting Information). Irrespective of NP size, reduction in attachment density restored the growth rate to the control bacteria levels. However, growth was unaffected for the smaller $40 \mathrm{~nm}$ gold NPs even at the high NP attachment density of 21 gold NPs $\mu^{-2}$ (Figure S5, Supporting Information).

Altogether, we posit that the new knowledge in the effect of assembly process parameters and linkage chemistry on bacteria-NP assembly outcomes and the effect of NP size on key bacterial behaviors of motility and growth in bacteria-based biohybrid systems will facilitate the design and development of more efficacious bacteria-mediated delivery systems for a variety of applications. The exact quantitative values for attachment density or changes in motility speed and growth rate are likely to depend on the choice of bacteria, NP, and linkage chemistry. Nonetheless, the observed trends, such as the effect of assembly parameters on NP attachment density and repeatability, are expected to be generalizable (Figure S5, Supporting Information). Similarly, the reported trends for the effect of linkage chemistry on NP attachment density or the effect of NP size and density on motility speed and growth rate can inform the design of process parameters and NP selection in other bacteria-based biohybrid systems.

\section{Experimental Section}

Bacteria Preparation: Salmonella Typhimurium VNP20009 che $Y^{+[45]}$ from a single colony was cultured overnight in MSB medium $\left(10 \mathrm{~g} \mathrm{~L}^{-1}\right.$ tryptone, $5 \mathrm{~g} \mathrm{~L}^{-1}$ yeast extract, $2 \mathrm{mM} \mathrm{MgSO}_{4}, 2 \mathrm{mM} \mathrm{CaCl}_{2}, \mathrm{pH} 7.0$ ) at $37^{\circ} \mathrm{C}$ and $100 \mathrm{rpm}$ (Excella E24 Incubator Shaker Series, New Brunswick Scientific). The overnight bacterial culture was diluted in MSB to $1 \% \mathrm{v} / \mathrm{v}$ and incubated at $37^{\circ} \mathrm{C}$ and $100 \mathrm{rpm}$ until an optical density at $600 \mathrm{~nm}\left(\mathrm{OD}_{600}\right)$ of 1.0 was reached. The bacterial culture was centrifuged at $1700 \times \mathrm{g}$ for $5 \mathrm{~min}$ twice and resuspended in motility buffer $\left(6.4 \mathrm{mM} \quad \mathrm{K}_{2} \mathrm{HPO}_{4}, \quad 3.5 \mathrm{mM} \quad \mathrm{KH}_{2} \mathrm{PO}_{4}, \quad 1 \mu \mathrm{M}\right.$ L-methionine, $10 \mathrm{mM}$ Sodium DL-lactate, $2 \mathrm{mM} \mathrm{MgSO}_{4}, 2 \mathrm{mM} \mathrm{CaCl}_{2}, \mathrm{pH}$ 7.0) to a final $\mathrm{OD}_{600}$ of 1.0 .

PLGA NP Synthesis: Pluronic F127 (100 mg) and deionized (DI) water $(20 \mathrm{~mL})$ were added to a glass vial. The vial was placed in a water bath sonicator (Branson 2510 Ultrasonic Cleaner, $100 \mathrm{~W}$ ) for $30 \mathrm{~min}$ to dissolve the Pluronic. Then, a magnetic stir bar was added, and the solution was stirred at $600 \mathrm{rpm}$. Acid-terminated PLGA $\left(M_{\mathrm{w}}: 25000 \mathrm{~g} \mathrm{~mol}^{-1}, 50: 50\right.$ lactic acid:glycolic acid, acid end-capped, Akina Inc. PolySciTech, West Lafayette, IN) was dissolved with dimethylformamide (DMF, SigmaAldrich, St. Louis, MO) to a final concentration of $22.22 \mathrm{mg} \mathrm{mL}^{-1}$; the solution was sonicated for $30 \mathrm{~min}$ to ensure molecular dissolution. While the PLGA solution was sonicating, 6,13-bis (triisopropylsilylethynyl) pentacene (TIPS, Sigma-Aldrich, St. Louis, MO) was dissolved in tetrahydrofuran (THF, anhydrous and uninhibited, $>99.9 \%$, Sigma-Aldrich, St. Louis, MO) to a final concentration of $3.05 \mathrm{mg} \mathrm{mL}^{-1}$. After the PLGA solution was fully dissolved, TIPS solution was added to the PLGA solution to achieve a 1:10 THF:DMF volume ratio. The mixture was vortexed for $\approx 5 \mathrm{~s}$ before loading into a $5 \mathrm{~mL}$ glass syringe with a $21 \mathrm{G}$ needle attached. Care was taken to remove all macroscopic air bubbles from the syringe. The TIPS:PLGA mixture $(1 \mathrm{~mL})$ was added dropwise $\left(0.5 \mathrm{~mL} \mathrm{~min}{ }^{-1}\right.$, NE-1000, New Era Pump Systems Inc.) to the stirring $(600 \mathrm{rpm})$ Pluronic F127 solution. The resulting NP suspension was allowed to stir for $5 \mathrm{~h}$ at $600 \mathrm{rpm}$. The suspension was protected from light to prevent degradation of the fluorophore. After $5 \mathrm{~h}$, the suspension was centrifuged at $22789 \times \mathrm{g}$ for $30 \mathrm{~min}$ at $4{ }^{\circ} \mathrm{C}$ (Sorvall Legend X1R, Thermofisher Scientific). The supernatant was discarded. The pellet was resuspended in $20 \mathrm{~mL}$ of $1 \times$ PBS by vortex mixing for $2 \mathrm{~min}$ and sonicating the suspension for $30 \mathrm{~min}$. The final dispersed suspension was passed through a nitrocellulose syringe filter $(0.45 \mu \mathrm{m}$ pore size) to remove any remaining aggregates. The filtrate was stored in a foil-wrapped vial at room temperature.

Streptavidin Functionalization of PLGA NPs: Microcentrifuge tubes were filled with $700 \mu \mathrm{L}$ of PLGA NP suspension. The tubes were centrifuged at $16060 \times g$ for $10 \mathrm{~min}$ at room temperature (accuSpin Micro, Fisher Scientific). The pellets were resuspended in $800 \mu \mathrm{L}$ of EDAC coupling solution $\left(20 \mathrm{mg} \mathrm{mL}^{-1}\right.$ EDAC, $5 \mu \mathrm{g} \mathrm{mL}^{-1}$ streptavidin-Cy3, pH $5.250 \mathrm{mM}$ MES buffer). The streptavidin coupling reaction took place on a vortex mixer (500 rpm, Fisher Digital Vortex 120 V, Fisher Scientific) for $3 \mathrm{~h}$. Following streptavidin coupling, the microcentrifuge tubes were centrifuged at $16060 \times \mathrm{g}$ for $10 \mathrm{~min}$ at room temperature. The pellets were resuspended in $100 \mu \mathrm{L}$ of motility buffer.

Dynamic Light Scattering Measurements: Dynamic light scattering (DLS) measurements were performed using a Zetasizer Nano ZS (Malvern Instruments) operating with Zetasizer Software v7.12. Disposable polystyrene cuvettes were filled with $1 \mathrm{~mL}$ of the final aqueous NP suspensions. Measurements were performed at room temperature, and the results are shown in Figure S6 and Table S1, Supporting Information.

Zeta Potential Measurements: The final aqueous NP suspensions were loaded into disposable polystyrene capillary cells. Zeta potential measurements were performed using a Zetasizer Nano ZS at room temperature. The results are shown in Figure S6 and Table S1, Supporting Information.

Nanoparticle Tracking Analysis: Dilutions (10x and 100x) of the functionalized NP suspensions were analyzed via nanoparticle tracking analysis (NTA) using a NanoSight NS500 (Malvern Instruments) operating with NanoSight NTA v3.4. All measurements were performed at room temperature. Five $1 \mathrm{~min}$ videos were taken for each sample, and the NP scattering cones were tracked to determine the number concentrations of the NP suspensions before incubating the NPs with bacteria to form NanoBEADS.

PLGA NanoBEADS Assembly: To prepare the ABS NanoBEADS, the prepared bacterial suspension in motility buffer was incubated with $10 \mu \mathrm{g} \mathrm{mL}^{-1}$ biotinylated Salmonella polyclonal antibody (Thermo Scientific, Waltham, MA, USA) on a vortex mixer at $500 \mathrm{rpm}$ at room temperature for $1 \mathrm{~h}$. The antibody-coated bacteria suspension was then centrifuged at $1700 \times g$ for 5 min to remove free antibody and suspended in motility buffer to an $\mathrm{OD}_{600}$ of 2.0 (Cary $60 \mathrm{UV}$-vis, Agilent Technologies). Next, the suspension of biotinylated antibody-coated bacteria was mixed with the streptavidin-coated NP at a bacteria to particles ratio of 1:100 in a volume of 100 or $800 \mu \mathrm{L}$ (as described in Table 1) and incubated on a vortex mixer at $500 \mathrm{rpm}$, a Belly Dancer mixer (IBI Scientific) at $100 \mathrm{rpm}$, or an end-over-end mixer (Fisher Scientific) at $15 \mathrm{rpm}$ for 30, 60 , or $90 \mathrm{~min}$ to facilitate the assembly of NPs onto the bacteria. For BS NanoBEADS, the bacterial suspension in the motility buffer was incubated with $0.4 \mathrm{mg} \mathrm{mL}^{-1}$ biotin (Fisher BioReagents, Fair Lawn, NJ) on a vortex mixer at $500 \mathrm{rpm}$ at room temperature for $30 \mathrm{~min}$ to physisorb 
biotin onto bacteria. Subsequently, the suspension was centrifuged at $1700 \times \mathrm{g}$ for $5 \mathrm{~min}$ to remove free biotin and resuspended the biotincoated bacteria in motility buffer to an $\mathrm{OD}_{600}$ of 2.0. Next, the suspension of bacteria coated with biotin was mixed with the streptavidin-coated NPs at a bacteria to particles ratio of 1:100 in a volume of $800 \mu \mathrm{L}$ and incubated on a Belly Dancer mixer at $100 \mathrm{rpm}$ or an end over end mixer at $15 \mathrm{rpm}$ for $60 \mathrm{~min}$. After the assembly process, the suspension of NanoBEADS was transferred to a centrifugal filter unit with a $0.8 \mu \mathrm{m}$ pore size high-flux polyethersulphone membrane (Sartorius Vivaclear, Elk Grove, IL) and centrifuged at $1700 \times \mathrm{g}$ for $30 \mathrm{~s}$ to remove free NPs. The NanoBEADS were suspended in motility buffer to an $\mathrm{OD}_{600}$ of 1.0 for NP areal density characterization experiments or in McCoy's 5A for the growth and motility assays.

NanoBEADS Samples Preparation for Field Emission Scanning Electron Microscope: To quantify the number of NPs attached to the outer membrane of bacteria, SEM images of NanoBEADS were taken using field emission scanning electron microscope (FE-SEM). A $10 \mu \mathrm{L}$ aliquot of the NanoBEADS suspension was deposited on $0.005 \% \mathrm{w} / \mathrm{v}$ poly-L-lysine (PLL)-treated glass slides and incubated at room temperature for $5 \mathrm{~min}$ to allow for attachment. Afterward, the slide was rinsed in DI water to remove the loosely attached NPs and NanoBEADS. Then, the slide was covered with $4 \%$ glutaraldehyde for $2 \mathrm{~h}$ at $4{ }^{\circ} \mathrm{C}$ to fix the attached NanoBEADS. Next, the slide was soaked in $0.1 \mathrm{M}$ phosphate-buffered saline (PBS) for 20 min twice. Finally, the same soaking process was repeated with DI water. After air-drying overnight, the slide was sputter-coated with $7 \mathrm{~nm}$ $\mathrm{Pt} / \mathrm{Pd}$ prior to imaging (Leica ACE600 sputter). High-resolution images were obtained utilizing a LEO (Zeiss) $1550 \mathrm{FE}-\mathrm{SEM}$ at an accelerating voltage of $5 \mathrm{kV}$ and working distances of $<8.6 \mathrm{~mm}$. To determine the average number of attached NPs for each NanoBEADS experiment, the particle numbers on $\approx 50$ bacteria were counted for each replicate. A minimum of two independent experiments were carried out for each case.

Viability Assay: The filtered NanoBEADS were diluted in motility buffer to an $\mathrm{OD}_{600}$ of 0.05 . To this $1 \mathrm{~mL}$ diluted NanoBEADS solution, $1.5 \mu \mathrm{L}$ aliquots of the $3.34 \mathrm{mM}$ SYTO 9 nucleic acid stain and $20 \mathrm{mM}$ propidium iodide (LIVE/DEAD BacLight Bacterial Viability Kit, Thermo Fisher, Eugene, OR, USA) were added, followed by incubation in the dark at room temperature for $15 \mathrm{~min}$ as per the manufacturer's instructions. The fluorescence microscopy images of NanoBEADS were taken using a Zeiss AxioObserver Z1 inverted microscope equipped with an AxioCam mRM camera at $40 \times$ objective. Live cells with an intact membrane stained green only, where cells with a damaged membrane or dead cells also stained red.

Growth Rate Measurement: The NanoBEADS suspensions were diluted in $3 \mathrm{~mL}$ of McCoy's $5 \mathrm{~A}$ medium supplemented with $10 \% \mathrm{FBS}$ to a final $\mathrm{OD}_{600}$ of 0.001 . The diluted NanoBEADS suspension was incubated at $37^{\circ} \mathrm{C}$ with shaking at $100 \mathrm{rpm}$ for $10 \mathrm{~h}$. A $100 \mu \mathrm{L}$ sample was taken every hour, diluted, and plated in triplicate on 1.5\% LB agar plates. Following overnight incubation at $37^{\circ} \mathrm{C}$, the bacteria colonies on the agar plates were counted to determine the NanoBEADS growth rate. A minimum of two independent experiments were carried out for each case.

Swimming Speed Measurement: The bacterial and NanoBEADS suspensions were diluted in $3 \mathrm{~mL}$ of McCoy's $5 \mathrm{~A}$ medium supplemented with $10 \%$ FBS to a final $O_{600}$ of 0.001 . The diluted suspensions were incubated at $37^{\circ} \mathrm{C}$ with shaking at $100 \mathrm{rpm}$ for $2 \mathrm{~h}$. A $10 \mu \mathrm{L}$ sample was taken, diluted, and placed on the glass coverslip. The videos of the bacteria movement were taken with a Zeiss AxioObserver Z1 inverted microscope equipped with an AxioCam $\mathrm{Hsm}$ camera and $63 \times$ oil immersion objective. The videos were analyzed in Image) using the MTrack) plugin tool. The average swimming speed was calculated by averaging the instantaneous speed, which is the traveled distance in each unit of time divided by the time unit. For each experiment, about 50 bacteria or NanoBEADS were tracked in the $15 \mathrm{~s}$ videos acquired at $32.8 \mathrm{fps}$. A minimum of two independent experiments were carried out for each case.

\section{Supporting Information}

Supporting Information is available from the Wiley Online Library or from the author.

\section{Acknowledgements}

This project was partially supported by the National Science Foundation (CAREER award, CBET-1454226) and the Institute for Critical Technology and Applied Science (ICTAS) at Virginia Tech.

\section{Conflict of Interest}

The authors declare no conflict of interest.

\section{Data Availability Statement}

The data that supports the findings of this study are available in the supplementary material of this article.

\section{Keywords}

bacteria-based cancer therapy, biohybrid robots, cell-mediated drug delivery systems, Salmonella enterica serovar Typhimurium, tumortargeting bacteria

Received: July 6, 2021

Revised: October 14, 2021

Published online:

[1] M. T. Stephan, J. J. Moon, S. H. Um, A. Bershteyn, D. J. Irvine, Nat. Med. 2010, 16, 1035.

[2] R. Mooney, L. Roma, D. Zhao, D. Van Haute, E. Garcia, S. U. Kim, A. J. Annala, K. S. Aboody, J. M. Berlin, ACS Nano 2014, 8, 12450.

[3] B. Huang, W. D. Abraham, Y. Zheng, S. C. B. López, S. S. Luo, D. J. Irvine, Sci. Transl. Med. 2015, 7, 291ra94.

[4] C. H. Villa, A. C. Anselmo, S. Mitragotri, V. Muzykantov, Adv. Drug Deliv. Rev. 2016, 106, 88

[5] M.-R. Choi, K. J. Stanton-Maxey, J. K. Stanley, C. S. Levin, R. Bardhan, D. Akin, S. Badve, J. Sturgis, J. P. Robinson, R. Bashir, N. J. Halas, S. E. Clare, Nano Lett. 2007, 7, 3759

[6] M. Roger, A. Clavreul, M. C. Venier-Julienne, C. Passirani, L. Sindji, P. Schiller, C. Montero-Menei, P. Menei, Biomaterials 2010, 31, 8393.

[7] D. Akin, J. Sturgis, K. Ragheb, D. Sherman, K. Burkholder, J. P. Robinson, A. K. Bhunia, S. Mohammed, R. Bashir, Nat. Nanotechnol. 2007, 2, 441.

[8] M. A. Traore, C. M. Damico, B. Behkam, Appl. Phys. Lett. 2014, 105, 173702.

[9] S. Suh, A. Jo, M. A. Traore, Y. Zhan, S. L. Coutermarsh-Ott, V. M. Ringel-Scaia, I. C. Allen, R. M. Davis, B. Behkam, Adv. Sci. 2019, 6, 1801309

[10] S. Xie, L. Zhao, X. Song, M. Tang, C. Mo, X. Li, J. Control. Release 2017 268, 390.

[11] C. K. Schmidt, M. Medina-Sánchez, R. J. Edmondson, O. G. Schmidt, Nat. Commun. 2020, 11, 1.

[12] J. Bastos-Arrieta, A. Revilla-Guarinos, W. E. Uspal, J. Simmchen, Front. Robot. Al 2018, 5, 97

[13] S. Gekle, Biophys. J. 2016, 110, 514.

[14] J. P. Celli, B. S. Turner, N. H. Afdhal, S. Keates, I. Ghiran, C. P. Kelly, R. H. Ewoldt, G. H. McKinley, P. So, S. Erramilli, R. Bansil, Proc. Natl. Acad. Sci. 2009, 106, 14321

[15] B. J. Toley, N. S. Forbes, Integr. Biol. (Camb). 2012, 4, 165.

[16] J. Adler, Science 1966, 153, 708.

[17] B. L. Taylor, I. B. Zhulin, M. S. Johnson, Annu. Rev. Microbiol. 1999 $53,103$. 
[18] D. Faivre, D. Schüler, Chem. Rev. 2008, 108, 4875.

[19] D. Bhaya, Mol. Microbiol. 2004, 53, 745.

[20] B. L. Taylor, D. E. Koshland Jr,, J. Bacteriol. 1975, 123, 557.

[21] M. Kihara, R. M. Macnab, J. Bacteriol. 1981, 145, 1209.

[22] C. Clairmont, K. C. Lee, J. Pike, M. Ittensohn, K. B. Low, J. Pawelek, D. Bermudes, S. M. Brecher, D. Margitich, J. Turnier, Z. Li, X. Luo, I. King, L. M. Zheng, J. Infect. Dis. 2000, 181, 1996.

[23] K. B. Low, M. Ittensohn, T. Le, J. Platt, S. Sodi, M. Amoss, O. Ash, E. Carmichael, A. Chakraborty, J. Fischer, S. L. Lin, X. Luo, S. I. Miller, L. Zheng, I. King, J. M. Pawelek, D. Bermudes, Nat. Biotechnol. 1999, 17,37

[24] M. Zhao, M. Yang, X.-M. Li, P. Jiang, E. Baranov, S. Li, M. Xu, S. Penman, R. M. Hoffman, Proc. Natl. Acad. Sci. U. S. A. 2005, 102, 755.

[25] J. F. Toso, V. J. Gill, P. Hwu, F. M. Marincola, N. P. Restifo, D. J. Schwartzentruber, R. M. Sherry, S. L. Topalian, J. C. Yang, F. Stock, L. J. Freezer, K. E. Morton, C. Seipp, L. Haworth, D. White, S. Macdonald, J. Mao, M. Sznol, S. A. Rosenberg, J. Clin. Oncol. 2002, 20, 142.

[26] D. M. Heimann, S. A. Rosenberg, J. Immunother. 2003, 26, 179.

[27] J. Nemunaitis, C. Cunningham, N. Senzer, J. Kuhn, J. Cramm, C. Litz, R. Cavagnolo, A. Cahill, C. Clairmont, M. Sznol, Cancer Gene Ther. 2003, 10, 737 .

[28] D. T. Le, T. W. Dubenksy, D. G. Brockstedt, Semin. Oncol. 2012, 39, 311.

[29] N. J. Roberts, L. Zhang, F. Janku, A. Collins, R.-Y. Bai, V. Staedtke, A. W. Rusk, D. Tung, M. Miller, J. Roix, K. V. Khanna, R. Murthy, R. S. Benjamin, T. Helgason, A. D. Szvalb, J. E. Bird, S. RoyChowdhuri, H. H. Zhang, Y. Qiao, B. Karim, J. McDaniel, A. Elpiner, A. Sahora, J. Lachowicz, B. Phillips, A. Turner, M. K. Klein, G. Post, L. A. Diaz, G. J. Riggins, N. Papadopoulos, K. W. Kinzler, B. Vogelstein, C. Bettegowda, D. L. Huso, M. Varterasian, S. Saha, S. Zhou, Sci. Transl. Med. 2014, 6, 249ral11.

[30] F. H. Schmitz-Winnenthal, N. Hohmann, T. Schmidt, L. Podola, T. Friedrich, H. Lubenau, M. Springer, S. Wieckowski, K. M. Breiner, G. Mikus, M. W. Büchler, A. V. Keller, R. Koc, C. Springfeld, P. Knebel, M. Bucur, L. Grenacher, W. E. Haefeli, P. Beckhove, Oncoimmunology 2018, 7, 1303584.

[31] D. T. Le, A. Wang-Gillam, V. Picozzi, T. F. Greten, T. Crocenzi, G. Springett, M. Morse, H. Zeh, D. Cohen, R. L. Fine, B. Onners, J. N. Uram, D. A. Laheru, E. R. Lutz, S. Solt, A. L. Murphy,
J. Skoble, E. Lemmens, J. Grous, T. Dubensky, D. G. Brockstedt, E. M. Jaffee, J. Clin. Oncol. 2015, 33, 1325.

[32] P. Basu, A. Mehta, M. Jain, S. Gupta, R. V. Nagarkar, S. John, R. Petit, Int. J. Gynecol. Cancer 2018, $28,764$.

[33] B. Behkam, M. Sitti, Appl. Phys. Lett. 2007, 90, 023902.

[34] M. C. M. van Loosdrecht, W. Norde, J. Lyklema, A. J. B. Zehnder, Aquat. Sci. 1990, 32, 103.

[35] O. Felfoul, M. Mohammadi, S. Taherkhani, D. de Lanauze, Y. Zhong Xu, D. Loghin, S. Essa, S. Jancik, D. Houle, M. Lafleur, L. Gaboury, M. Tabrizian, N. Kaou, M. Atkin, T. Vuong, G. Batist, N. Beauchemin, D. Radzioch, S. Martel, Nat. Nanotechnol. 2016, 11, 941.

[36] S. Taherkhani, M. Mohammadi, J. Daoud, S. Martel, M. Tabrizian, ACS Nano 2014, 8, 5049.

[37] R. Kazmierczak, E. Choe, J. Sinclair, A. Eisenstark, Methods Mol. Biol. 2015, 1225, 151.

[38] V. Du Nguyen, J.-W. Han, Y. J. Choi, S. Cho, S. Zheng, S. Y. Ko, J.-O. Park, S. Park, Sensors Actuators B Chem. 2015, 224, 217.

[39] O. Yasa, M. Sitti, Y. Alapan, O. Schauer, V. Sourjik, A. F. Tabak, J. Giltinan, Sci. Robot. 2018, 3, eaar4423.

[40] X. Xu, Y. Chen, H. Wei, B. Xia, F. Liu, N. Li, Anal. Chem. 2012, 84, 9721.

[41] M. Kojima, Z. Zhang, M. Nakajima, T. Fukuda, Biomed. Microdevices 2012, 14, 1027.

[42] S. Suh, E. J. Leaman, Y. Zhan, B. Behkam, in Proc. 40th Annu. Int. Conf. IEEE Eng. Med. Biol. Soc., IEEE, Piscataway, NJ 2018, pp. 6162-6165.

[43] S. Song, M. S. Vuai, M. Zhong, Infect. Agent. Cancer 2018, 13, 9.

[44] S. Zhou, C. Gravekamp, D. Bermudes, K. Liu, Nat. Rev. Cancer 2018, 18, 727.

[45] K. M. Broadway, S. Suh, B. Behkam, B. E. Scharf, J. Biotechnol. 2017, 251, 76.

[46] V. Huter, M. P. Szostak, J. Gampfer, S. Prethaler, G. Wanner, F. Gabor, W. Lubitz, J. Control. Release 1999, 61, 51.

[47] N. Buss, O. Yasa, Y. Alapan, M. B. Akolpoglu, M. Sitti, APL Bioeng. 2020, 4, 026103.

[48] Y. Hiratsuka, M. Miyata, T. Uyeda, Biochem. Biophys. Res. Commun. 2005, 331, 318.

[49] A. V. Singh, Z. Hosseinidoust, B. W. Park, O. Yasa, M. Sitti, ACS Nano 2017, 11, 9759.

[50] R. W. Carlsen, M. R. Edwards, J. Zhuang, C. Pacoret, M. Sitti, Lab Chip 2014, 14, 3850. 